\title{
THREE-MANIFOLDS ADMITTING METRICS WITH THE SAME GEODESICS
}

\author{
VladimiR S. MATVEeV
}

\begin{abstract}
If two different metrics on a closed three-manifold have the same geodesics, then the manifold is homeomorphic to a lens space or to a Seifert manifold with zero Euler number.
\end{abstract}

\section{Introduction}

Definition 1. Let $g$ be a Riemannian metric on a manifold $M^{n}, n \geq 2$. A Riemannian metric $\bar{g}$ on $M^{n}$ is called geodesically equivalent to $g$, if any geodesic of $\bar{g}$, considered as unparameterized curve, is a geodesic of $g$.

For example, the proportional metrics $g$ and $C \cdot g$, where $C$ is a non-zero constant, are geodesically equivalent. Note that if the metrics are conformally equivalent and geodesically equivalent, then the conformal coefficient is constant so that they are proportional [4].

Theorem 1. A closed connected three-dimensional manifold admits nonproportional geodesically equivalent Riemannian metrics if and only if it is homeomorphic either to a lens space or to a Seifert manifold with zero Euler number.

A two-dimensional analogue of Theorem 1 is:

Theorem 2 (Matveev, Topalov [9]). A closed surface admits nonproportional geodesically equivalent Riemannian metrics if and only if its Euler characteristic is non-negative.

Theorems 1 and 2 tell us that the existence of nonproportional geodesically equivalent metrics yields simple topological restrictions on $M^{n}, n=2,3$. For dimensions greater than three, such restrictions are expected to be sufficiently more complicated. One can show that every finitely-presented group can be realized as the fundamental group of a closed manifold admitting nonproportional geodesically equivalent metrics. The latter is not true, if we assume in addition that there exists a point where the eigenvalues of one metric with respect to the other are all different.

Received October 15, 2001.

Research at INIMS was supported by EPSRC grant GRK99015. 
Theorem 3 (Matveev, Topalov). If a closed connected manifold admits geodesically equivalent Riemannian metrics $g$ and $\bar{g}$ such that the eigenvalues of the tensor $G_{i}^{j} \stackrel{\text { def }}{=} g_{i \alpha} \bar{g}^{\alpha j}$ are all different at least at one point of the manifold, then the manifold can be covered by the product of spheres.

We will neither prove nor explain this theorem in the present paper. A proof will appear elsewhere, in a joint paper with Topalov.

1.1. History. Beltrami [1] was the first to observe that two different metrics can have the same geodesics. At the end of his paper [1], he formulated the problem of describing all geodesically equivalent metrics on surfaces. It is not clear from the text whether he assumed the local or the global description; actually, his motivation came from a certain problem of cartography which requires a global setting. Nevertheless, since Dini [3], Levi-Civita [6], Weyl [16], E. Cartan [2] and Eisenhart [4], the theory of geodesically equivalent metrics was mostly a local geometry.

Global aspects of geodesic equivalence have been intensively studied in 50th and later. But, probably because of the influence of earlier researchers, all known global results require additional strong geometrical assumptions. Roughly speaking, one takes some geometric condition written in tensor form (i.e., the metric is assumed to be Kählerian or semisymmetric or generally semisymmetric or T-generalized semisymmetric or Einsteinian or of constant curvature or locallyproduct or almost Hermitian or Ricci-flat or recurrent or admitting a concircular vector field or admitting a torse-forming vector field), combines it with a tensor reformulation of geodesic equivalence and deduces new tensor objects with global geometric properties, see the survey paper [12].

\section{Integrability of geodesic flows for geodesically equivalent metrics}

Let $g=\left(g_{i j}\right)$ and $\bar{g}=\left(\bar{g}_{i j}\right)$ be Riemannian metrics on $M^{n}$. Consider the (1,1)-tensor $L$ given by the formula

$$
L_{j}^{i} \stackrel{\operatorname{def}}{=}\left(\frac{\operatorname{det}(\bar{g})}{\operatorname{det}(g)}\right)^{\frac{1}{n+1}} \bar{g}^{i \alpha} g_{\alpha j} .
$$

Then, $L$ determines the family $S_{t}, t \in R$, of $(1,1)$-tensors

$$
S_{t} \stackrel{\text { def }}{=} \operatorname{det}(L-t \mathrm{Id})(L-t \mathrm{Id})^{-1} .
$$

Let us identify the tangent and cotangent bundles of $M^{n}$ by $g$. This identification allows us to transfer the natural Poisson structure from $T^{*} M^{n}$ to $T M^{n}$.

Theorem 4 ([8, 10]). If $g, \bar{g}$ are geodesically equivalent, then, for every $t_{1}, t_{2} \in$ $R$, the functions

$$
I_{t_{i}}: T M^{n} \rightarrow R, \quad I_{t_{i}}(\xi) \stackrel{\text { def }}{=} g\left(S_{t_{i}}(\xi), \xi\right)
$$

are commuting integrals for the geodesic flow of $g$. 
At every point $x \in M^{n}$, let us denote by

$$
\lambda_{1}(x) \leq \lambda_{2}(x) \leq \ldots \leq \lambda_{n}(x)
$$

the eigenvalues of $L$ at the point $x$.

Corollary 1. Let $\left(M^{n}, g\right)$ be a geodesically complete connected Riemannian manifold. Let Riemannian metric $\bar{g}$ on $M^{n}$ be geodesically equivalent to $g$. Then, for every $i \in\{1, \ldots, n-1\}$, for every $x, y \in M^{n}$, the following holds:

1. $\lambda_{i}(x) \leq \lambda_{i+1}(y)$.

2. If $\lambda_{i}(x)<\lambda_{i+1}(x)$, then $\lambda_{i}(z)<\lambda_{i+1}(z)$ for almost every point $z \in M^{n}$.

3. If $\lambda_{i}(x)=\lambda_{i+1}(y)$, then there exists $z \in M^{n}$ such that $\lambda_{i}(z)=\lambda_{i+1}(z)$.

Proof of Corollary 1 for dimension three. Note that the restriction of the integrals $I_{t}$ to the tangent space to $x \in M^{3}$ depends on the restriction of the metrics to $T_{x} M^{3}$ only. On the tangent space to $x$, we can always find "diagonal" coordinates $\xi_{1}, \xi_{2}, \xi_{3}$ such that in these coordinates $g$ is Euclidean and $L$ is diagonal. Then, the integrals $I_{t}(x, \xi)$ are given by

$$
\left.I_{t}=\left(\lambda_{2}-t\right)\left(\lambda_{3}-t\right) \xi_{1}^{2}+\left(\lambda_{1}-t\right)\left(\lambda_{3}-t\right) \xi_{2}^{2}+\left(\lambda_{1}-t\right)\left(\lambda_{2}-t\right)\right) \xi_{3}^{2} .
$$

In order to prove Corollary 1 , we need the following simple lemma. For every $(x, \xi) \in T M^{3}$, denote by $t_{1}(x, \xi) \leq t_{2}(x, \xi)$ the roots of the polynomial $I_{t}(x, \xi)$.

Lemma 1 ([11]). The following holds:

1. For every $\xi \in T_{x} M^{3}$,

$$
\lambda_{1}(x) \leq t_{1}(x, \xi) \leq \lambda_{2}(x) \leq t_{2}(x, \xi) \leq \lambda_{3}(x) .
$$

2. If $\lambda_{i}(x)<\lambda_{i+1}(x)$, then, for every $\tau \in R$, the Lebesgue measure of the set

$$
V_{\tau} \subset T_{x} M^{3}, \quad V_{\tau} \stackrel{\text { def }}{=}\left\{\xi \in T_{x} M^{3}: t_{i}(x, \xi)=\tau\right\},
$$

is zero.

The first statement of Corollary 1 follows immediately from the first statement of Lemma 1: Consider a geodesic $\gamma: R \rightarrow M^{3}, \gamma(0)=x, \gamma(1)=y$. By Theorem 4 , every root $t_{i}$ is constant on every orbit $(\gamma, \dot{\gamma})$ of the geodesic flow of $g$ so that

$$
t_{i}(\gamma(0), \dot{\gamma}(0))=t_{i}(\gamma(1), \dot{\gamma}(1)) .
$$

Using Lemma 1, we obtain

$$
\lambda_{i}(\gamma(0)) \leq t_{i}(\gamma(0), \dot{\gamma}(0)), \quad \text { and } \quad t_{i}(\gamma(1), \dot{\gamma}(1)) \leq \lambda_{i+1}(\gamma(1)) .
$$

Hence, $\lambda_{i}(\gamma(0)) \leq \lambda_{i+1}(\gamma(1))$ and the first statement of Corollary 1 is proved.

Let us prove the second statement of Corollary 1. Suppose $\lambda_{i}(y)=\lambda_{i+1}(y)=$ $\tau$ for every point $y$ of $V \subset M^{3}$. Note that, by the first statement of Corollary 1 , $\tau$ is a constant (independent of $y \in V$ ). Take a point $x \in M^{3}$. Let us prove that $\lambda_{i}(x)=\lambda_{i+1}(x)=\tau$. Let us join the point $x$ with every point of $V$ by all possible geodesics. Consider the set $V_{\tau} \subset T_{x} M^{3}$ of the initial velocity vectors (at the point $x$ ) of these geodesics. 
By the first statement of Lemma 1, for every geodesic $\gamma$ passing through at least one point of $V$, the value $t_{i}(\gamma, \dot{\gamma})$ is equal to $\tau$. Then, by the second statement of Lemma 1, the measure of $V_{\tau}$ is zero. Since $V$ lies in the image of the exponential mapping of $V_{\tau}$, the measure of $V$ is also zero. The second statement of Corollary 1 is proved.

Let us prove the third statement of Corollary 1. Let $\lambda_{i}(\gamma(0))=\lambda_{i+1}(\gamma(1))=$ $\lambda$ for a certain $i \in\{1,2\}$ and for some constant $\lambda$. We will assume that $\lambda_{i}(\gamma(0))<$ $\lambda_{i+1}(\gamma(0))$. Let us show that the geodesic $\gamma$ consists of points where $\lambda_{i}$ or $\lambda_{i+1}$ are equal to $\lambda$.

If $t_{i}$ is a multiple root of the polynomial $I_{t}(\gamma(0), \dot{\gamma}(0))$, then the statement obviously follows from Lemma 1 . If $\lambda_{i-1}(\gamma(0))=\lambda$, the statement follows from the first statement of Corollary 1.

Suppose $t_{i}$ is not a multiple root and either $i=1$ or $\lambda_{i-1}(\gamma(0))<\lambda$. Consider the function $I_{\lambda}: T M^{3} \rightarrow R$. Let us show that the differential $d I_{\lambda}$ vanishes at every point $(\gamma(\tau), \dot{\gamma}(\tau))$. Evidently, the differential of an integral is preserved by the geodesic flow so that it is sufficient to prove that the differential vanishes at the point $(\gamma(0), \dot{\gamma}(0))$.

By Lemma 1, we have

$$
\lambda=\lambda_{i}(\gamma(0)) \leq t_{i}(\gamma(0), \dot{\gamma}(0))=t_{i}(\gamma(1), \dot{\gamma}(1)) \leq \lambda_{i+1}(\gamma(1))=\lambda .
$$

Hence, $\lambda$ is a root of the polynomial $I_{t}(\gamma(0), \dot{\gamma}(0))$ and $I_{\lambda}(\gamma(0), \dot{\gamma}(0))=0$. By assumptions, the eigenvalue $\lambda_{i}$ has multiplicity one in a small neighborhood of $\gamma(0)$. Then, it is a smooth function on this neighborhood, and the function $I_{\lambda_{i}(z)}(z, \nu)$ is also smooth on the tangent bundle to this neighborhood. Consider the function $I_{\lambda}(z, \nu)-I_{\lambda_{i}(z)}(z, \nu)$. Its differential vanishes at the point $(\gamma(0), \dot{\gamma}(0))$. More precisely, by assumptions, $\lambda$ is a simple root of the polynomial $I_{t}(\gamma(0), \dot{\gamma}(0))$ so that in a neighborhood of the point $(\lambda,(\gamma(0), \dot{\gamma}(0))) \in R \times T M^{3}$ the function $I_{t}(z, \nu)$ is a monotone function in $t$. But $\lambda_{i}$ is not greater than $\lambda$. Then, the difference $I_{\lambda}(z, \nu)-I_{\lambda_{i}(z)}(z, \nu)$ is either always non-positive or always non-negative in a small neighborhood of $(\gamma(0), \dot{\gamma}(0))$. At the point $(\gamma(0), \dot{\gamma}(0))$, we have $\lambda_{i}(\gamma(0))=\lambda$. Then, $I_{\lambda}(\gamma(0), \dot{\gamma}(0))-I_{\lambda_{i}(\gamma(0))}(\gamma(0), \dot{\gamma}(0))=0$. Hence, the function $I_{\lambda}(z, \nu)-I_{\lambda_{i}(z)}(z, \nu)$ has a local extremum at $(\gamma(0), \dot{\gamma}(0))$. Finally, its differential vanishes at the point.

The differential of the function $I_{\lambda_{i}(z)}(z, \nu)$ also vanishes at the point $(\gamma(0), \dot{\gamma}(0))$. More precisely, combining (1) and the first statement of Corollary 1 , we obtain that the function $I_{\lambda_{i}}$ is either always non-positive or always non-negative. But $I_{\lambda_{i}(\gamma(0))}(\gamma(0), \dot{\gamma}(0))$ is equal to $I_{\lambda}(\gamma(0), \dot{\gamma}(0))$ and is zero. Then, the point $(\gamma(0), \dot{\gamma}(0))$ is an extremum of the function $I_{\lambda_{i}}$ and, therefore, the differential of $I_{\lambda_{i}}$ vanishes at the point $(\gamma(0), \dot{\gamma}(0))$.

Thus the differential of the function $I_{\lambda}$ vanishes at every point of the curve $(\gamma, \dot{\gamma})$. Then, the partial derivatives $\frac{\partial I_{\lambda}}{\partial \xi_{\alpha}}$ given by

$$
2\left(\lambda-\lambda_{2}\right)\left(\lambda-\lambda_{3}\right) \xi_{1}, 2\left(\lambda-\lambda_{1}\right)\left(\lambda-\lambda_{3}\right) \xi_{2}, 2\left(\lambda-\lambda_{1}\right)\left(\lambda-\lambda_{2}\right) \xi_{3}
$$


are equal to zero. Thus $\lambda_{i}=\lambda$ or $\lambda_{i+1}=\lambda$ at every point of the geodesic. Finally, there exists a point of the geodesics where $\lambda_{i}=\lambda_{i+1}$. Corollary 1 is proved.

\section{Ideas and main steps of proof for Theorem 1}

By Corollary 1, only the following four cases are possible:

1. There exist $\tau_{1}<\tau_{2}$ such that for every $x \in M^{3}$

$$
\lambda_{1}(x) \leq \tau_{1} \leq \lambda_{2}(x) \leq \tau_{2} \leq \lambda_{3}(x),
$$

and there exist $x_{1}, x_{2} \in M^{3}$ such that $\lambda_{1}\left(x_{1}\right)=\lambda_{2}\left(x_{1}\right), \lambda_{3}\left(x_{2}\right)=\lambda_{2}\left(x_{2}\right)$.

2. The eigenvalue $\lambda_{2}$ is constant, and there exist $x_{1}, x_{2}, x_{3} \in M^{3}$ such that $\lambda_{1}\left(x_{1}\right)=\lambda_{2}, \lambda_{1}\left(x_{2}\right)<\lambda_{2}<\lambda_{3}\left(x_{2}\right)$ and $\lambda_{3}\left(x_{3}\right)=\lambda_{2}$.

3. At every $x \in M^{3}$, at least two eigenvalues of $L$ are equal, and there exists a point where all eigenvalues are equal.

4. There exists $\tau \in R$ such that either $\lambda_{1}(x)<\tau<\lambda_{2}(x)$ for every $x \in M^{3}$ or $\lambda_{2}(x)<\tau<\lambda_{3}(x)$ for every $x \in M^{3}$.

3.1. Case 1. In this case, for different $t_{1}, t_{2}, t_{3}$, the integrals $I_{t_{1}}, I_{t_{2}}, I_{t_{3}}$ are functionally independent almost everywhere and the geodesic flow of $g$ is Liouvilleintegrable. Note that the integrals are quadratic in velocities and simultaneously diagonalizable at every point of the manifold. Then, $g$ is Liouville in the sense of Kiyohara [5], page 1. One can prove that $g$ is proper (in the sense of [5], page 2 ) if and only if every eigenvalue of $L$ is not constant, and that the rank (in the sense of [5], page 3) of $g$ is equal to one. Then, by Theorem 3.3.1 from [5], the manifold $M^{3}$ is homeomorphic either to $S^{3}$ or to $R P^{3}$.

It appears that it is possible to generalize the methods of [5] to all geodesically equivalent metrics satisfying assumptions of Case 1. As a result, one can prove that the manifold $M^{3}$ is homeomorphic to a lens space. Differ from the case of proper Liouville metrics, every lens space possesses nonproportional geodesically equivalent metric. New topological possibilities are closely related to the following two-dimensional observation: Consider a Riemannian metric on a surface such that its geodesic flow is Liouville-integrable, the integral is quadratic in velocities, the integral and the Hamiltonian are proportional at the tangent space to a point $x$ of the surface. Consider the group $G$ of the isometries preserving the point $x$ and the integral. If no non-trivial linear combination of the Hamiltonian and of the integral is the square of a function linear in velocities, then $G$ has maximum two elements. If the integral is a square of a function linear in velocities, then $G$ is infinite and is isomorphic to $O(2)$.

3.2. Case 2. We will prove Theorem 1 modulo the Poincare conjecture only. A proof that does not require the Poincare conjecture is rather lengthy and will appear elsewhere.

First of all, there exists a point of the manifold where $\lambda_{1}=\lambda_{2}=\lambda_{3}$. Moreover, every geodesic $\gamma$ such that $\lambda_{1}(\gamma(0))=\lambda_{2}=\lambda_{3}(\gamma(1))>\lambda_{1}(\gamma(1))$ consists of 
points where $\lambda_{1}=\lambda_{2}$ or $\lambda_{2}=\lambda_{3}$ and, therefore, contains a point where $\lambda_{1}=$ $\lambda_{2}=\lambda_{3}$.

The proof is similar to the proof of the third statement of Corollary 1, the role of the function $I_{\lambda}$ plays the function

$$
I_{\lambda_{2}}^{\prime} \stackrel{\text { def }}{=}\left(\frac{d}{d t} I_{t}\right)_{\mid t=\lambda_{2}} .
$$

By Lemma 1 and Theorem $4, \lambda_{2}$ is a double-root of the polynomial $I_{t}(\gamma, \dot{\gamma})$. Then, for every point $(\gamma(\tau), \dot{\gamma}(\tau))$ of the geodesic, we have $I_{\lambda_{2}}(\gamma(\tau), \dot{\gamma}(\tau))=0$, $I_{\lambda_{2}}^{\prime}(\gamma(\tau), \dot{\gamma}(\tau))=0$ and $d I_{\lambda_{2}}^{\prime}(\gamma(\tau), \dot{\gamma}(\tau))=0$. Combining these conditions, we obtain that $\lambda_{1}(\gamma(\tau))=\lambda_{2}$ or $\lambda_{3}(\gamma(\tau))=\lambda_{2}$. Thus there exists a point where all eigenvalues of $L$ are equal. Suppose the number of such points is greater than four. Then, every point $x$ of a certain open non-empty subset of the manifold is a transversal intersection of at least three geodesics passing through at least one point where $\lambda_{1}=\lambda_{2}=\lambda_{3}$. Let us show that this is impossible.

By Lemma 1, the integrals $I_{\lambda_{2}}, I_{\lambda_{2}}^{\prime}$ vanish on every geodesic passing through a point where $\lambda_{1}=\lambda_{2}=\lambda_{3}$. By Corollary 1, without loss of generality, we can assume that $\lambda_{1}(x)<\lambda_{2}(x)<\lambda_{3}(x)$. Then, the set

$$
\left\{\xi=\left(\xi_{1}, \xi_{2}, \xi_{3}\right) \in T_{x} M^{3}: I_{\lambda_{2}}(x, \xi)=0, I_{\lambda_{2}}^{\prime}(x, \xi)=0\right\}
$$

is organized in two (intersected at zero) straight lines. Therefore three geodesics passing through points where $\lambda_{1}=\lambda_{2}=\lambda_{3}$ can not be mutually transversal at $x$. This contradiction proves that the number of the points where $\lambda_{1}=\lambda_{2}=\lambda_{3}$ is not greater than four.

Actually, the number of points where $\lambda_{1}=\lambda_{2}=\lambda_{3}$ is always even. More precisely, in view of (1), the integral $I_{\lambda_{2}}$ is (at least locally) the square of an integral, linear in velocities. Evidently, the integral vanishes at the tangent space to a point if and only if $\lambda_{1}=\lambda_{2}$ or $\lambda_{2}=\lambda_{3}$ at the point. Since the zeros of a Killing vector field on a three-manifold are organized into one-dimensional closed submanifolds, the set

$$
M \stackrel{\text { def }}{=}\left\{x \in M^{3}:\left(\lambda_{1}(x)-\lambda_{2}\right)\left(\lambda_{3}(x)-\lambda_{2}\right)=0\right\}
$$

is a one-dimensional submanifold. Since it is closed and connected, it is homeomorphic to the circle. The points $x$ such that $\lambda_{1}(x)=\lambda_{2}=\lambda_{3}(x)$ divide this circle into intervals. For every point of each interval, either $\lambda_{3}>\lambda_{2}$ or $\lambda_{1}<\lambda_{2}$. Consider a point $x$ where $\lambda_{1}(x)=\lambda_{2}=\lambda_{3}(x)$, and two such intervals having $x$ as an endpoint. Clearly, $\lambda_{3}>\lambda_{2}$ on one of this interval and $\lambda_{1}<\lambda_{2}$ on the other. More precisely, take a convex neighborhood $U(x)$ of diameter less than the radius of injectivity, a point $y \in U$ such that $\lambda_{1}(y)<\lambda_{2}<\lambda_{3}(y)$, and all geodesics in $U$ connecting all the points of $M \cap U$ with the point $y$. Clearly, the velocity vectors at the point $y$ of all these geodesics lie on the plane orthogonal to the eigenvector of $L$ corresponding to $\lambda_{1}$. Consider the restriction of the function $I_{\lambda_{2}}^{\prime}$ to this plane. Its zeros are organized into two straight lines intersecting at the point $(0,0)$. The initial velocity vector of the geodesic lying in $U(x)$ and 
passing through the point $x$ lies on one of this line. The velocity vectors of the geodesics coming from the points of one interval lie from one side of the straight line, the velocity vectors of the geodesics coming from the points of the other interval lie from the other side of the straight line.

Evidently, the function $I_{\lambda_{2}}^{\prime}$ is positive from one side of the line and negative from the other. Hence, $\lambda_{3}>\lambda_{2}$ on one of these intervals and $\lambda_{1}<\lambda_{2}$ on the other.

Finally, the number of points where $\lambda_{1}=\lambda_{2}=\lambda_{3}$ is either two or four. Then, the fundamental group of the manifold is either trivial or isomorphic to $Z_{2}$. In the first case, modulo the Poincare conjecture, the manifold is homeomorphic to the sphere. In the second case, modulo the Poincare conjecture and in view of [7], the manifold is homeomorphic to $R P^{3}$.

3.3. Case 3. We will show that the number of points where $\lambda_{1}=\lambda_{2}=\lambda_{3}$ is no greater than two. Then, modulo the Poincare conjecture and in view of [7], the manifold is homeomorphic to the sphere or to the projective space.

Suppose there exist three points where $\lambda_{1}=\lambda_{2}=\lambda_{3}$. Without loss of generality, we can assume that at every point of the manifold $\lambda_{2}=\lambda_{3}=$ const. Consider the integral $I_{\lambda_{2}}^{\prime}$ given by (2). The value of this integral on every geodesic passing through a point where $\lambda_{1}=\lambda_{2}=\lambda_{3}$ is equal to zero. Then, the velocity vectors $\dot{\gamma}$ of such geodesics are eigenvectors of the tensor $L$ with the eigenvalue $\lambda_{1}$. Then, two such geodesics can not intersect transversally in a regular point, and, therefore, the number of the points where $\lambda_{1}=\lambda_{2}=\lambda_{3}$ is maximum two.

3.4. Case 4. Without loss of generality, we can assume that there exists a constant $\tau$ such that $\lambda_{1}(x)<\tau<\lambda_{2}(x)$ for every $x \in M^{3}$. Consider the $(1,1)$ tensor $D$ given by

$$
D \stackrel{\text { def }}{=}\left(L^{2}-\left(\operatorname{trace}(L)-\lambda_{1}(x)\right) L+\frac{\operatorname{det}(L)}{\lambda_{1}(x)} \operatorname{Id}+L-\lambda_{1}(x) \operatorname{Id}\right)^{-1} .
$$

The above condition on the eigenvalues of $L$ insures that the tensor $D$ is welldefined, is self-adjoint with respect to $g$, and all its eigenvalues are positive. Consider the Riemannian metric $h_{i j} \stackrel{\text { def }}{=}\left(g_{i \alpha} D_{j}^{\alpha}\right)$.

At every point of the manifold, there exist coordinates $x_{1}, x_{2}, x_{3}$ such that the vector $\frac{\partial}{\partial x_{1}}$ is the eigenvector of $L$ with the eigenvalue $\lambda_{1}$, and $h$ is given by

$$
d s_{h}^{2}=d x_{1}^{2}+B\left(x_{2}, x_{3}\right)\left(d x_{2}^{2}+d x_{3}^{2}\right),
$$

where the function $B$ depends on the variables $x_{2}, x_{3}$ only.

Indeed, it is sufficient to verify this statement at almost every point of the manifold. By Corollary 1, almost every point has a neighborhood where the number of different eigenvalues of $L$ is constant. In such neighborhoods, the statement follows from the local description of geodesically equivalent metrics given by the classical Levi-Civita theorem [6], which can be found in most classical textbooks on differential geometry. 
Thus the Riemannian manifold $\left(M^{3}, h\right)$ is locally the product of two- and one-dimensional manifolds, and the components of the product are invariantly defined. Then, at least on the double-cover of the manifold, we can find a vector field such that it is Killing with respect to $h$ and the distribution orthogonal to this vector field is integrable. Note that in view of $[13,14]$ and $[15]$, it is sufficient to show that this double-cover is Seifert with no Euler number. We can assume that the orbits of this vector field are closed. More precisely, the flow of the vector field acts by isometries; hence it generates a one-parametric subgroup in the compact Lie group of all isometries of the manifold. The closure of this subgroup is closed and commutative. Hence, it is a Lie subgroup isomorphic to the torus. We can evidently find a one-dimensional closed Lie subgroup in this torus in such a way that the corresponding Killing vector field does not vanish.

Hence, the manifold is Seifert. Since, locally, the section of the Seifert foliation can be given invariantly (as the two-dimensional surface orthogonal to our Killing vector field), it is possible to construct a global section. Hence, the Euler number of the manifold equals zero.

\section{Examples of geodesically equivalent metrics on the lens spaces and on Seifert manifolds with zero Euler number}

In order to construct nonproportional geodesically equivalent metrics on the lens spaces, we use the following three-dimensional generalization of Beltrami's example from [1]. Consider the real four-dimensional space $R^{4}$ with the complex coordinates $z_{1}=x_{1}+i y_{1}, z_{2}=x_{2}+i y_{2}$, the sphere

$$
S^{3}=\left\{\left(z_{1}, z_{2}\right) \in R^{4}:\left|z_{1}\right|^{2}+\left|z_{2}\right|^{2}=1\right\} \subset R^{4} .
$$

We denote by $g$ the restriction of the Euclidean metric $d s^{2}=d z_{1} d \bar{z}_{1}+d z_{2} d \bar{z}_{2}$ to the sphere. Consider the linear transformation of $R^{4}$ given by $A:\left(z_{1}, z_{2}\right) \mapsto$ $\left(a_{1} z_{1}, a_{2} z_{2}\right)$, where $a_{1} \neq a_{2}$ are positive constants. Denote by $\bar{g}$ the pull-back $l^{*} g$, where the mapping $l: S^{3} \rightarrow S^{3}$ is given by $l: v \mapsto \frac{A(v)}{|A(v)|}$.

The metrics $g$ and $\bar{g}$ are geodesically equivalent. Indeed, the geodesics of $g$ are great circles (the intersections of planes that go through the origin with the sphere). The mapping $A$ is linear and, therefore, takes planes to planes. Since the normalization $w \mapsto \frac{w}{|w|}$ takes planes to their intersections with the sphere, the mapping $l$ takes great circles to great circles. Evidently, $A$ is not proportional to an orthogonal transformation. Hence, the metrics $g$ and $\bar{g}$ are not proportional.

Recall that a lens space is the quotient space of $S^{3}$ modulo the action of the group $Z_{q}$ generated by

$$
\left(z_{1}, z_{2}\right) \mapsto\left(e^{2 \pi \frac{1}{p} i} z_{1}, e^{2 \pi \frac{q}{p} i} z_{2}\right)
$$

for the appropriate mutually-prime $p$ and $q$. Since the action preserves $g$ and $\bar{g}$, the metrics generate a pair of nonproportional geodesically equivalent metrics on the lens space. 
In order to construct geodesically equivalent Riemannian metrics on a closed Seifert manifold with zero Euler number, we use the following trivial observation: for every Riemannian manifolds $\left(M_{1}, g_{1}\right)$ and $\left(M_{2}, g_{2}\right)$, the metrics $g_{1}+g_{2}$ and $g_{1}+2 \cdot g_{2}$ are geodesically equivalent on the direct product $M_{1} \times M_{2}$.

It is known [13], that every closed Seifert manifold with zero Euler number can be constructed by the following procedure. Take a closed surface $F^{2}$, a finite group $H$ acting on $F^{2}$, the group $Z_{p}$ (and its standard action on $S^{1}$ generated by $\left.\phi \mapsto \phi+\frac{2 \pi}{p}\right)$ and a subgroup $G$ of the group $H \times Z_{p}$ such that the natural action of the subgroup $G$ on the product $F^{2} \times S^{1}$ is free. The quotient space is a closed Seifert manifold with zero Euler number, and every closed Seifert manifold with zero Euler number can be obtained using this construction.

Since the group $H$ is finite and, hence, compact, there exists a metric $g_{2}$ on $F^{2}$ invariant with respect to the action of $H$. Denote by $g_{1}$ the metric $d \phi^{2}$ on $S^{1}$. The Riemannian metrics $g_{1}+g_{2}$ and $g_{1}+2 \cdot g_{2}$ are invariant modulo the action of the group $G$ and, therefore, induce nonproportional geodesically equivalent metrics on the quotient space.

\section{Acknowledgements}

I am grateful to Prof. V. Bangert, Prof. A. Bolsinov, Prof. M. Igarashi, Prof. K. Kiyohara, Prof. S. Matveev and Prof. P. Topalov for useful discussions. I would like to thank The European Post-Doctoral Institute for partial financial support and The Isaac Newton Institute for Mathematical Sciences for hospitality.

\section{References}

[1] E. Beltrami, Resoluzione del problema: riportari $i$ punti di una superficie sopra un piano in modo che le linee geodetische vengano rappresentante da linee rette, Ann. Mat. 1 (1865), no. 7, 185-204.

[2] E. Cartan, Lecons sur la theorie des espaces a connexion projective. Redigees par $P$. Vincensini, Gauthier-Villars., Paris, 1937.

[3] U. Dini, Sopra un problema che si presenta nella theoria generale delle rappresetazioni geografice di una superficie su un'altra, Ann. Mat. Ser. 2, 3 (1869), 269-293.

[4] L. P. Eisenhart, Riemannian Geometry. 2d printing, Princeton University Press, Princeton, NJ, 1949.

[5] K. Kiyohara, Two classes of Riemannian manifolds whose geodesic flows are integrable, Mem. Amer. Math. Soc. 130 (1997), no. 619.

[6] T. Levi-Civita, Sulle trasformazioni delle equazioni dinamiche, Ann. di Mat., serie $2^{a}$, 24 (1896), 255-300.

[7] G. R. Livesay, Fixed point free involutions on the 3-sphere, Ann. of Math. (2) 72 (1960), 603-611.

[8] V.S. Matveev, P. J. Topalov, Trajectory equivalence and corresponding integrals, Regul. Chaotic Dyn. 3 (1998), no. 2, 30-45.

[9] _ Metric with ergodic geodesic flow is completely determined by unparameterized geodesics, Electron. Res. Announc. Amer. Math. Soc. 6 (2000), 98-104 (electronic).

[10] Geodesic equivalence and integrability, preprint, 1998.

[11] Vladimir S. Matveev, Geschlossene hyperbolische 3-Mannigflatigkeiten sind geodätisch starr, Manuscripta Math. 105 (2001), no. 3, 343-352. 
[12] J. Mikes, Geodesic mappings of affine-connected and Riemannian spaces. Geometry, 2., J. Math. Sci. 78 (1996), no. 3, 311-333.

[13] P. Scott, The geometries of 3-manifolds, Bull. London Math. Soc. 15 (1983), no. 5, 401487.

[14] (1983), no. 1, 35-70.

[15] J. L. Tollefson, The compact 3-manifolds covered by $S^{2} \times R^{1}$, Proc. Amer. Math. Soc. 45 (1974), 461-462.

[16] H. Weyl, Zur infinitisimalgometrie: Einordnung der projectiven und der konformen Auffasung, Nachrichten von der K. Gesellschaft der Wissenschaften zu Göttingen, Mathematisch-Physikalische Klasse, 99-112, 1921; "Selecta Hermann Weyl," Birkhäuser Verlag, Basel und Stuttgart, 1956.

Mathematisches Institut, Universität Freiburg, 79104 Germany.

E-mail address: matveev@email.mathematik.uni-freiburg.de 